\title{
A SURVEY OF ARTIFICIAL INTELLIGENCE TECHNIQUES EMPLOYED FOR ADAPTIVE EDUCATIONAL SYSTEMS WITHIN E-LEARNING PLATFORMS
}

\author{
Khalid Almohammadi $^{1}$, Hani Hagras ${ }^{1}$, Daniyal Alghazzawi ${ }^{2}$, Ghadah Aldabbagh ${ }^{2}$ \\ ${ }^{1}$ The Computational Intelligence Centre, \\ School of Computer Science and Electronic Engineering University of Essex, Colchester, UK \\ ${ }^{2}$ Faculty of Computing and Information Technology, \\ King Abdulaziz University Jeddah, Saudi Arabia
}

\begin{abstract}
The adaptive educational systems within e-learning platforms are built in response to the fact that the learning process is different for each and every learner. In order to provide adaptive e-learning services and study materials that are tailor-made for adaptive learning, this type of educational approach seeks to combine the ability to comprehend and detect a person's specific needs in the context of learning with the expertise required to use appropriate learning pedagogy and enhance the learning process. Thus, it is critical to create accurate student profiles and models based upon analysis of their affective states, knowledge level, and their individual personality traits and skills. The acquired data can then be efficiently used and exploited to develop an adaptive learning environment. Once acquired, these learner models can be used in two ways. The first is to inform the pedagogy proposed by the experts and designers of the adaptive educational system. The second is to give the system dynamic self-learning capabilities from the behaviors exhibited by the teachers and students to create the appropriate pedagogy and automatically adjust the e-learning environments to suit the pedagogies. In this respect, artificial intelligence techniques may be useful for several reasons, including their ability to develop and imitate human reasoning and decision-making processes (learning-teaching model) and minimize the sources of uncertainty to achieve an effective learning-teaching context. These learning capabilities ensure both learner and system improvement over the lifelong learning mechanism. In this paper, we present a survey of raised and related topics to the field of artificial intelligence techniques employed for adaptive educational systems within e-learning, their advantages and disadvantages, and a discussion of the importance of using those techniques to achieve more intelligent and adaptive e-learning environments.
\end{abstract}

Keywords: e-learning, adaptive educational systems, artificial intelligence

\section{Introduction}

The enhancement of student learning performance and satisfaction represents one of the main objectives of educational systems. In order to be able to tailor the teaching process according to the needs and preferences of each student, teachers have to conduct accurate evaluations of the different competencies of students, which can naturally differ in terms of level of knowledge, interest, so- 
cial background, and level of motivation [1], [2]. An expert teacher in the classroom environment is aware of the differentiated characteristics and learning abilities of the students. However, there are limits to the degree to which any teacher can adjust the learning environment to optimally educate every student simultaneously due to classroom size and the accuracy of the evaluation process conducted by the teacher [1]. Therefore, the accuracy of learning and analysing such characteristics can be facilitated by a smaller class size, which would allow teachers to focus on the needs and preferences of each individual student [1]. Studies have shown that, in contrast to group education, one-to-one teaching is more likely to generate a higher student learning performance [3], [4], [5]. However, it might be difficult to provide such attention and teaching in traditional classrooms.

The Internet has become a central core to the educative environment experienced by learners, thus facilitating learning at any location and at any time [6]. Allen and Seaman [7] claimed that in 2008 , nearly a quarter of all students in postsecondary and further education in the USA were taking courses delivered exclusively online. By 2009, Ambient Insight Research reported that 44\% of post-secondary students in the USA were taking at least some of their courses online and projected that penetration would increase to $81 \%$ by 2014 [8]. Thus, developed economies have been the principle market for self-paced e-learning products in recent times [9]. However, developing economies are now enthusiastically embracing e-learning due to the huge increase in suppliers [9].

A global e-learning system is beginning to define itself. In 2011, the market for self-paced elearning reached a total of $\$ 35.6$ billion worldwide and has a five-year compound growth rate of $7.6 \%$ [9]. By 2016, revenues will be as high as $\$ 51.5$ billion (as shown in Figure 1) [9]. Such findings across the world, and in the USA in particular, reflect the global rapid adoption of e-learning, from an emergent alternative to traditional course delivery. Hence, e-learning is rapidly entering mainstream and becoming a predominant method for delivering post-secondary education [8], [10].

E-learning can be viewed as a system of electronic learning whereby instructions are devised or formatted to support learning and then delivered to the intended beneficiaries through digital devices that normally come in the form of computers or mobile devices [11]. E-learning may be designed in two forms where one form of e-learning is designed as an instructor-led type of learning known as synchronous e-learning, while the other is designed in a format that is a self-paced individual study, known as asynchronous e-learning as is shown in Figure 2 [11]. In asynchronous e-learning, when the learners take up a course study that utilizes spoken or printed texts that come in the form of illustrations, photos, animation, or video as learning materials, and with which evaluations are made, the learners are then given the opportunity to control the time and place as well as the pace at which they want to undertake their own learning [11], [12]. The other elearning format, known as synchronous e-learning, is real-time instructor-led training that is designed for instructions on the learning to be delivered or facilitated by an instructor to take place in a real time [11], [13]. Generally, different communication tools are utilized for this type of e-learning format, which is usually delivered in real time, mostly over the Internet. Students undertaking the training usually $\log$ on at a scheduled time and establish communications directly with the instructors [13]. Unfortunately, it appears that these e-learning environments, which could be asynchronous learning environments or synchronous learning environments, have the same problems raised in normal classrooms due to the lack of interaction, which means that the diagnosing process cannot be fully applied between the teachers and students. In addition, the e-learning courses are offered and designed for all students, without considering the individual students' unique needs and abilities [14], [15].

In adaptive educational systems, the learner characteristics are monitored and the instructional milieu is appropriately adjusted to offer support and to make improvements to the learning process [17], [18]. Such systems are receiving much interest as a result of their ability to deliver instructional content and analysis by actively adapting to the individual student requirements and needs [17], [19]. Adaptive educational systems contain three different models. The learner profile or model is used as means to infer and diagnose student abilities and characteristics, the taught model content representation will be learnt, and the instructional model is used to convey and match how the con- 


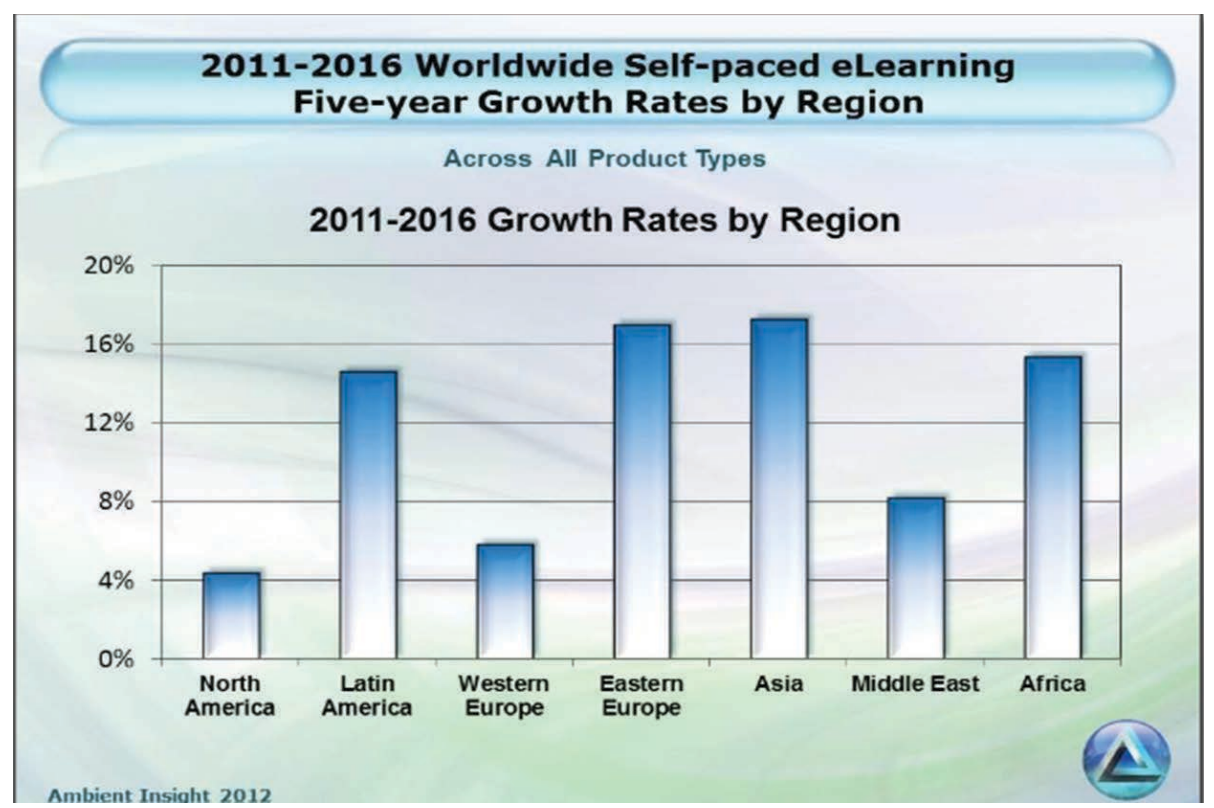

Figure 1. Worldwide Self-paced Five-year Growth Rates by Region [9]

tent is suggested to the learner in adaptive and dynamic contexts [18]. The efficiency of adaptive educational systems depends on the methodology employed to collect and diagnose information regarding the learning needs and characteristics of students as well as how this information is processed to develop an adaptive and intelligent learning context [17]. Student needs and characteristics in the teaching environment can be classified based on many variables, such as current student knowledge, learning styles, affective states, personality traits, and student goals [14]. The main objective of considering these variables is to allow students to better achieve their learning goals and objectives [20]. Course content could be adapted to each learner through feedback, content sequencing, and the presentation of materials in different teaching style approaches [17]. In this paper, we present a review of the some of the raised related topics to the adaptive educational system and associated artificial intelligence techniques, and their advantages and disadvantages, along with the importance of utilizing those techniques to achieve more intelligent and adaptive learning environments.

In Section 2, we will present an overview of some recent topics related to the adaptive educational system. Section 3 will give an overview of the artificial intelligence techniques employed for adaptive educational systems. Section 4 will present recommendations for future research and a conclusion.

\section{Overview of Recent Topics Re- lated to AI techniques for Adap- tive Educational Systems}

Adaptive educational systems emphasize the importance of individual differences in modelling the ideal online learning environment. Identifying and catering for individual learners' requirements and capabilities is key to success in the provision of adaptive e-learning systems. Adaptive educational systems must have these skills if they are to provide suitable learning methods and content to their users [17]. Therefore, it is critical to create accurate student profiles and model based on the analysis of their affective states, level and type of knowledge, personality traits and skills. This data must then be efficiently used to develop adaptive learning environment [17]. The acquired data must then be efficiently used and exploited to develop adaptive learning environment. Once acquired, these learner models and data can be used in two ways. The first is to inform the pedagogy proposed by the experts and designers of the adaptive educational system. The second is to give the system dynamic selflearning capabilities from teachers' or students' behavior. These learning capabilities will ensure both learner and system improvement over the lifelong learning mode.

Educational data mining and learner analytics are two major overlapping fields that could be very 
Live (synchronous) (asynchronous) On Demand

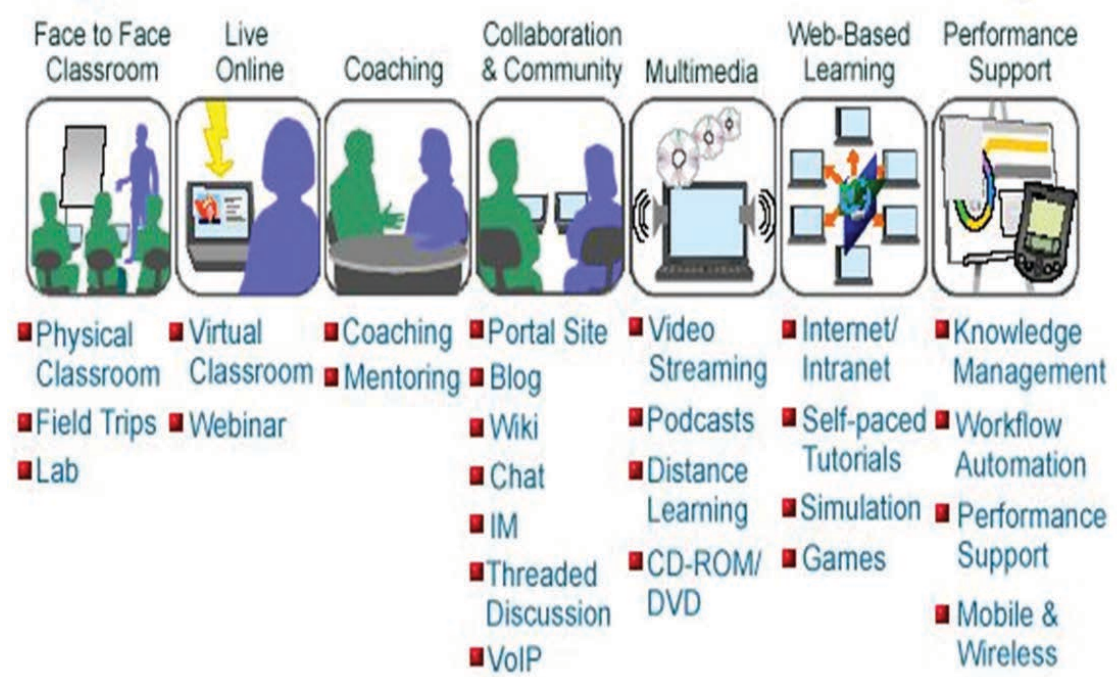

Figure 2. Asynchronous and synchronous learning settings

influential in originating such capabilities. Artificial intelligence models are also useful in achieving such capabilities, as they are capable of developing and imitating the human decision-making process. It is therefore necessary to review and examine in further detail the present and related topics to adaptive learning environments, such as massive open online courses (MOOCs), educational data mining, learner analytics, and associated artificial intelligence techniques.

\subsection{Massive Open Online Courses}

MOOCs represent a concept that became known in 2008. This type of open learning system has rapidly become popular [21]. Two features must be present in any MOOC: it must be free of cost and open to any user [22]. Recently, some leading universities and colleges, including Stanford and Harvard, have made MOOCs available for learning [22]. The number of users that may be enrolled in any course can reach from thousands to millions - that is, they can have a massive number of users [22].

The main challenge regarding MOOCs lies in collecting enormous data sets from students' interactions with their learning environments and combining them with artificial intelligence techniques to create insight into adaptive human learning [21]. If we can use these techniques to predict the success, the next important step could be to use analyt- ics to power personalized systems that are adaptive to students' preferences and needs based on the collected behaviours of learners as well as that of past students' data sets [21]. According to [23], there are high dropout rates in the number of students who enrolled within massive open online courses, and the completion rates in those courses are less than $13 \%$. Adaptive educational learning driven by learner behaviour analysis could be the solution. Learning interaction analyses are not just beneficial for academic reasons; they also help students obtain feedback on their performance and learning styles [24]. However, despite the capabilities of learning analysis technologies and tools, there is a need for human interpretation of the data [24]. Fauvel and Yu [25] have published a detailed survey about artificial intelligence techniques and data mining applications for MOOCs.

\subsection{Educational data mining and learner analytics}

Various Learning Management Systems (LMS) examples of which are: Sakai, Moodle and ILIAS or portals dealing with learning like Dreambox, Knewton or massive open online courses can deliver e-learning courses [26]. These courses have the standout feature of availability of huge amount of data that is available in a compiled form. Every step of the process is dictated by the student's personal attributes and evaluation of his performance [26]. 
Two fields of research are dedicated to analysis of this data: Learning Analytics (LA) and Educational Data Mining (EDM) [26].The description of EDM and LAK groups share similar similarities. According to The International Educational Data Mining Society, EDM is described as a new field of study that aims to prepare techniques for exploration of specialized form of information received from the educational sector and the application of these techniques to improve understanding about students and the environment in which they imbibe knowledge [27]. According to The Society for Learning Analytics Research, Learning Analytics (LA) are defined as the compilation, quantification, analysis and notification of information related to students in relation to their individual characteristics so that the process of learning can be well understood and improved upon along with the surroundings in which it takes place [27]. The extreme popularity of such methods is specifically attributed to the following factors:

- Just like in business studies or analytical fields there is a growing trend for using a technique based on analysis of data in order to arrive on improved decisions [26], [28].

- There is availability of strong machine learning, statistical and data mining techniques and procedures which are able to identify similarities in data and develop decision rules and predictive models which are easy to use in context of information compiled from the education sector.

- Compilation of facts and information has become simpler and the modern day computers have enough memory to process and store the information.

- Due to the budgetary constraints and increasing competition educational institutions have been forced to cut down costs and generate more revenue by tapping into the growing educational market of the developing nations, motivating students to continue with their courses and providing improved standard of services [26].

Both LA and EDM have the main purpose of searching for relevant information from data obtained from educational sector to provide backing to decisions related to this sector. The facts may be presented so as to cater to the needs of the various parties with an interest in the process [26], [29]. The response or feedback obtained helps the educators to analyze the content of the study material and the success of the learning process as well. If a continuous watch is kept on the performance of the students then any student facing problems in grasping the course material can be detected at the earliest instance and this can also help to pin pointing specific parts of the course that are causing a greater difficulty. If the proper methods are not available then the entire process becomes more difficult and will take a longer time [26], [30]. Students will have the facility of being guided through the appropriate resources in accordance with their aims and needs, motivation and performance, they can visualize the results of the learning in graphical form and can see where they stand in comparison to fellow class members and also visualize the efforts and performance in context of coeducational pursuits. In a nut shell, it can be said that the systems can be used in different ways within the framework of an adaptive educational system as follows:

- Modeling of behaviour patterns of users, their knowledge levels and their experiences,

- Profiling of users,

- Modelling of important principles of any field and also modelling the knowledge components of a domain,

- Analytical study of trends. The use of analytics to modify the process in accordance with or to suit the preference and needs of the user is another area where such techniques can be applied [31].

But, the main stumbling block in successful use of LA and EDM methods is limited awareness and knowledge [26], a large number of educators and authorities lack knowledge in both practical functioning and theoretical concepts as far as the correct use of these tools is concerned, they are unable to correctly analyse the results, decipher the inferences correctly or take a decision regarding the steps to be taken. To find a solution to this problem, an effort must be made to create a positive environment for and cultivate a data centred approach (data driven approach) in the educational 
sector [32]. [33], [34], [35] and enumerate the surveys and vision conducted in context of learning analytical studies and educational data mining and their application for educational objectives and in relation for creating adaptive e-learning courses .

\subsubsection{Educational data Mining techniques}

AI (largely involving machine learning) and statistical tools are the two techniques that DM primarily utilizes. There are two broad categories to which the DM methods can be classified [25]:

- Predictive, which can be further split to, a) sequential prediction and interpolation, and, b) supervised learning

- Descriptive, which is split into: a) clustering, and, b) exploratory analysis

\subsubsection{Predictive Methods}

These methods are used to obtain single or multiple variables with predicted value from predictor variable or group of variables. It is divided in three types [36].

- Classification- this is used to predict class label in form of perpetuate or discrete. The most commonly used classification approaches in EDM make use of, decision trees and logistic regression [36].

- Regression- this is utilised to derive prediction from continuous variables. The most common regression approaches for EDM are neural networks and linear regression [36].

- Prediction of density where predicted values are derived by using probability density function. Many different kernel functions can be used in EDM to estimate the value of density including Gaussian functions [36].

\subsubsection{Association Rule Mining}

The purpose of association rule mining is to derive the If-Then rules which work in a manner that if a certain collection of variables is present then a different variable is bound to have a certain value. An example of this rule is given below [37]:
IF a student feels a sense of frustration OR has a strong desire to learn instead of just delivering a good result THEN he will seek assistance often [37].

Rules mined using this approach uncover common occurrences in data which would have been difficult to discover manually. When this technique is used, then the rule framed helps in detecting frequent coincidental patterns in the data which otherwise are hard to detect on manual analysis [37].

As far as EDM are considered, association rules mining has been studied in great detail. The interrelationship between various factors present in data is detected and if-then declarations are prepared in context of these factors [38], [39]. Two different lines of thought have led to the adoption of association rules mining to e- learning methods: The first is that the teachers find it easier to receive proper feedback of the web based learning methods i.e. understanding how students imbibe knowledge through the Internet, assess students by studying their navigation through the site, divide the student in groups, redesign the website so as to individualise the course material, the second beneficial effect is that the students can receive assistance regarding working on an electronic set up, an example of this is the modification in the study material as per the student's performance by using information collected from past students to suggest the correct learning techniques for a particular student [40].

\subsubsection{Clustering Methods}

Grouping of similar objects together is known as clustering. Use of clustering methods can help in identification of objects in a particular region as dense or few and the pattern of description can be understood with detection of interrelationship among various data parameters. Classification techniques can also help in correct differentiation of various varieties of objects but it's an expensive process hence clustering is used as an initial approach for choosing subsets of parameters and further classification. 


\section{An Overview on Artificial Intel- ligence Methodologies Employed for Adaptive Educational Sys- tems}

The aim of adaptive educational systems is to tailor the overall learning approach in order to fulfil the needs of students [15]. Hence, it is essential that the profiles and models of students are created accurately with consideration for the examination of their affective states, levels of knowledge, skills, and personality traits. The information required then needs to be utilised and developed in order to improve the adaptive learning environment [15]. Hence, AI approaches are regarded as valuable tools, as they have the ability to develop and replicate the decision-making process adopted by people [42]. There are various AI techniques that have been used in adaptive educational systems, such as Fuzzy Logic (FL), Decision tree, Bayesian networks, Neural Networks, Genetic algorithms, and Hidden Markov Models. There are various ways through which AI approaches are used in adaptive educational systems. For example, in some systems, the core focus is to examine and assess student characteristics to generate profiles of the students with the intention of evaluating their overall level of knowledge to be used as basis for prescribed software pedagogy [43], [44], [45], [46], [47], [48], [49]. The AI approaches are also used to facilitate the diagnostic process completion so that course content can be adjusted to cater to the needs of every student, and some of them are used to learn from the student behaviours to adjust the prescribed software pedagogy [50], [51], [52], [53], [47], [54].

However, most of the existing adaptive educational systems do not learn from student behaviours. Adaptive educational systems that depend on the ideas of a few experts or designers that are used in tackling student behaviour might be characterised by various sources of uncertainty about the learner response evaluation with an adaptive educational system, linked to learner reception of instruction. Various sources of uncertainty can occur in e-learning environments, resulting from examining student variables, such as assessments or the engagement level. In addition, needed instructional action outcomes, such as what concept should be studied in accordance with this assessment and en- gagement, combine in a suitable form of proposed environment targets to recognise ideal learning activities. This form of learning-teaching decision is often needed to deal with information that is uncertain (we are not sure that the available information is absolutely true) and/or imprecise (the values handled are not completely defined) [55]. An example of a rule that we need to deal with would be: 'if the student knowledge in Excel is very low and in PowerPoint is high, then he/she should study moderate Excel materials'. We are not sure that this rule and each antecedent and consequent is absolutely true for the target learners. Therefore, how do we ensure high accuracy in assessing the individual's knowledge level, learning style, and other needs in order to provide the best and correct individual adaptive action? This question is quite critical, due to several sources of uncertainties in how accurately student responses are actually assessed by adaptive educational methods as well as the corresponding uncertainties associated with how the resulting instruction to the student is actually understood and received. In e-learning environments, there are high levels of linguistic uncertainties, where the individual students can differ greatly in how the same terms, words, or methods (e.g., course difficulty or length of study time) are received and comprehended, which can vary according to student motivation, knowledge, and future plans about learning a given subject in an e-learning environment.

Relying on designer or expert knowledge for guiding the pedagogy of the adaptive educational system may be considered time-consuming and costly. Furthermore, it may even be impossible to tackle the varied characteristics of learners in some cases due to incomplete knowledge about what constitutes effective instruction. In proposing a pedagogy, automatic learning from learner behaviors can make the design of adaptive e-learning and teaching system more convenient and effective, which saves the effort and time of experts and designers. Even more, it will give them insight into what makes online instruction effective. The learning models generated from student behaviors can be easily edited and modified in a lifelong learning model. Thus, developing adaptive educational systems based on the knowledge of how learners interact with the learning environment in readable and interpretable white box models is critical in the guidance of the adaptation approach for learner 
needs as well as understanding the way learning is achieved. The AI techniques, such as FL, decision trees, neural networks, genetic algorithms, and Markov models, can manage the inherent uncertainty that human decision making has, and they are innovative approaches that can deal with impression, uncertainty, and partial truth. In this respect, these AI techniques are useful for several reasons, including that they are capable of developing and imitating the human decision-making process and building automatic and accurate teaching-learning models [56].

\subsection{White box approaches}

\subsubsection{Fuzzy logic}

Fuzzy logic was initially presented by Zadeh in 1965 [57] where it quickly became a popular and efficient technique for user modeling, as it could imitate human reasoning [58]. Fuzzy logic can be seen as an extension for the traditional set theory as statements can be partial truths, lying in between absolute truth and absolute falsity [59]. The Fuzzy Logic System (FLS) comprises four stages (as shown in Figure 3) which are: fuzzifier, rule base, inference engine, and defuzzifier [59]. Rules can be extracted from numerical data or supplied by experts. Upon the establishment of rules, an FLS may be considered as a mapping from the crisp inputs to crisp outputs, such mapping may be articulated numerically as $y=f(x)$ [59].

Learning-teaching behaviour is represented in a human readable and linguistically interpretable manner by the fuzzy rules. Their transparency makes them perfect to be assessed quickly to explain the reason and method of certain combinations of input-actuated specific rules, where a certain set of output conclusions has been yielded. There is an association with linguistic labels appearing in the consequent and antecedent of rules grouped as input and output values in the system. Adapting the fuzzy sets of FLS is possible from the data that is generated from the system and the student interactions.

A new category and extension of fuzzy systems can be defined as type 2 FLSs, where type 2 fuzzy sets are used to convey numeric and linguistic uncertainty [60]. This type 2 fuzzy system can be proposed to directly model and reduce the effects of uncertainties [60]. An extended part of type $1 \mathrm{FL}$ is known as type $2 \mathrm{FL}$, and type $2 \mathrm{FL}$ will be minimised to type $1 \mathrm{FL}$ with complete disappearance of uncertainty [60]. There are more degrees of freedom in a type-2 FLS compared to the type-1 FLSs [61]. Type 2 FLSs provide a methodology for tackling different sources of numeric and linguistic uncertainty that exist in e-learning environments.

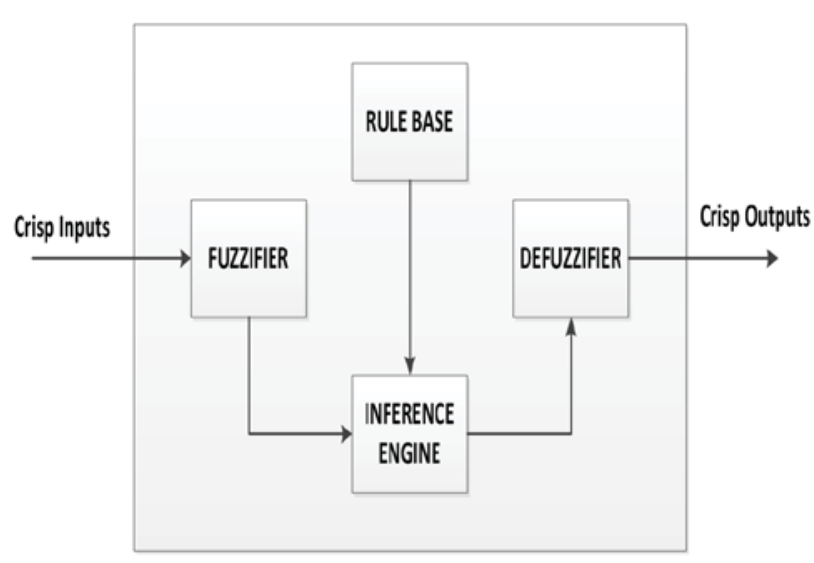

Figure 3. Block diagram of a Fuzzy Logic System [60]

A framework geared towards user-modelling, based on the FLS, induces simplified reasoning for both users and designers, which therefore assists in terms of amendments and comprehension [56], [62], [63]. Furthermore, FLSs are commonly utilized in order to examine and assess learningand knowledge-related outcomes [64], [65], [46], [66], [48], [49], [67]. More precisely, FLSs can be adopted in order to evaluate and examine task objectives as well as multiple criteria assessments, as demonstrated by previous researchers [64], [65], [48],[ 66], [48], [49], [67]. The FLS are also used to facilitate the diagnostic process completion, known as student modelling, so that course content can be adjusted to cater to the needs of every student. In relation to $\mathrm{Xu}$ [54], a profiling system adopting a multi-agent approach has been presented, whereby the creation of fuzzy models for content and students was based on a dynamic plan formally defined ahead of time for one individual. This framework was obtained through profile abstraction, which is recognized as comprising student-centred learning tasks, such as the topic at hand and the time spent on the topic. Furthermore, the content framework was devised and created with fuzzy links between 
the subjects, and the knowledge of the individuals (referred to as prerequisite relations) were established to be utilized in order to formally determine the learning adaptation (i.e., the order of issues to be examined by the individual) [54]. The work of [68] employs FL to model user knowledge of domain concepts. The work represents the dependencies between domain concepts in order to cycle graph, as some concepts have essential or supportive perquisites between them, and they use fixed rules to accomplish dynamic updating of user knowledge regarding the concepts. Through these procedures, the right concepts are adapted to the students.

The research proposed by [69], [70] and [71] employs a self-learning mechanism that generates a FL-based model from the data. It also incorporated and gauged the student engagement levels, and mapped them to suitable delivery needs, which match the knowledge and preferred learning styles of the students. The proposed practical and theoretical environments incorporated a novel system for gauging the student engagement levels based on utilizing visual information to automatically calculate the engagement degree of the students. This differs from traditional methods that usually employ expensive and invasive sensors. This approach only used a low-cost Red Green Blue-Depth (RGBD) video camera (Microsoft Kinect) operating in a non-intrusive mode, whereby the users were allowed to act and move without restrictions. This fuzzy model is generated from data representing various student capabilities and their desired learning needs. The learnt type- 1 and typ- 2 models was then used to improve the knowledge delivery to the various students based on their individual characteristics. The proposed environment was adaptive, where it was continuously adapting in a lifelong learning mode to ensure that the generated models adapted to the individual student needs and preferences. This employed approach was not computationally demanding and generated easily read and analyzed white box models, which can be checked by the lay user, which is mainly suitable for adapting the dynamic nature of the e-learning process.

In [72], a system based on type 2 FL utilizing visual RGB-D features was presented, including head pose direction and facial expressions captured from Kinect (v2), a low-cost but robust 3D camera, to measure the engagement degree of students in both remote and onsite education. This system augmented another self-learning type 2 FLS that helps teachers with recommendations of how to adaptively vary their teaching methods to suit the level of students and enhance their instruction delivery. This proposed dynamic e-learning environment integrates both onsite and distance students as well as teachers who instructed both groups of students. The rules were learnt from the student and teacher learning/teaching behaviors, and the system was continuously updated to give the teacher the ability to adapt the delivery approach to varied learner engagement levels.

\subsubsection{Decision Trees}

A decision tree is a tree in which each branch node represents a choice between a number of alternatives, and each leaf node represents a decision [73], [74]. Decision tree are commonly used for gaining information for the purpose of decision making [73], [75]. Decision tree starts with a root node which is for users to take actions. From this node, users split each node recursively according to decision tree learning algorithm. The final result is a decision tree in which each branch represents a possible scenario of decision and its outcome [73]. Importantly, decision-tree techniques has been proposed to be effective in ensuring that individual requirements are met and in improving the efficiency of learning, especially in the context of e-learning [76], [77].

In their case, Lin et al. [77] presented a system for personalized learning paths in which decisiontree techniques inform the creativity e-Learning system. Whereas, Kumar Baradwaj et. al. [78] utilised the decision tree technique ID3 in exploring the data set that contained student data collected over a three-year period. In identifying the most appropriate attribute on the basis of Information Gain, Previous Semester Mark (PSM) attribute is established to be the basis of the decision tree. After the collected data has been classified several IF-THEN rules are devised and corroborated with the decision tree established to provide insights regarding the performance of students and provide the necessary resources to students requiring special attention. According to Adhatrao et. al. [79], the application of decision-tree techniques can be used in predict- 
ing student performance and they went ahead to use Iterative Dichotomiser 3 (ID3) as well as C4.5 classification algorithms based on RapidMiner (a data mining tool) to predict performance of the students. In their attempts at predicting student performance, Mrinal Pandey et. al. [80] utilized Reptree, Nave Bayes and Simple cart by means of WEKA mining tool. Another study [81] advanced an adaptive system analysis that can be relied upon to optimize learning sequences. This analysis utilizes a decision tree model based on students' profiles to establish the learning system that would be most adaptive for a specific teaching content. By utilizing the decision tree methods, supervised learning is considered to be the foundation and the information gain theory is applied to establish the attributes to be utilized in constructing a minimal-attributes decision tree. Nevertheless, these tend to be unsatisfactory because of their inability to adequately deal with special and extreme situations [82], [83].

\subsection{Black box approaches}

\subsubsection{Neural networks}

Neural Networks are more commonly becoming used to model human behavior, and therefore replicate human actions and responses [42]. Fausett [84] and Haykin [85] provide good overviews of what Neural Networks are and how they operate [42]. In essence, a Neural Network [NN] comprises a large amount of interwoven neurons or component parts which work together to process information and solve problems. In effect, it is a system which takes in and analyses information much akin to biological nervous systems, for example, the brain. NNs do not require any information about a particular issue before resolving it [42]. They can process information and produce results which are far more complex than other information processing paradigms and therefore render them a very influential means of modeling human conduct [42]. Therefore, they are an effective AI method which has mainly been proposed in many scientific areas, but also the educational field. In the educational field, their popularity has stemmed from their ability to classify students, share characterizations, and simulate and track learners 'cognitive progress [86], [42]. They have several relevant skills; amongst these is the ability to learn in an adaptive way and furthermore to recognize matching in comparable situations. They recognize patterns and are able to extract meaning from imprecise or unclear source data [86].

A considerable number of researches have used the Neural Network method in the context of adaptive educational system. A system built for the purpose of predicating the performance of MBA students prior to registration with a particular institute was proposed by Naik [87]. The results of this system, used to separate pupils into successful and secondary units were far superior than those of other statistical processes in terms of reliability and promise. The results of Ibrahim's system [88] and Idris [51], which used a Neural Network to forecast student's academic results, also showed more potential than others. Another use of Neural Networks consists of identifying sets of learning objectives and, further to that, which learning objects which are most suited to a specific person. What is most suited refers to the likeness of the precedents which represent the domain concept of the profile of the student and the make-up of the learning objective. This initial work demonstrates that a teacher's duties and rule-based choices can be replicated by an adaptation model created using this computational intelligence method [12]. This work introduced in [12] presents the works that have been recently developed regarding concept-based categorization of learning objects based on artificial neural network (ANN). The study employed Back Propagation (BP) and Self Organizing Map (SOM) algorithms to establish the connection that domain concepts of the learning object have with the learning needs of the learner to provide the learning object that is appropriate for each user.

Nevertheless, the black-box features of Neural Networks are difficult to align with the modeling of human behavior due to the representation of knowledge, the weights learned are often difficult for humans to interpret [47].

\subsubsection{Bayesian networks}

Bayesian networks are a widely used method for student modeling in intelligent learning systems [89]. A Bayesian Network (BN) is a Direct Acyclic Graph (DAG), that is, a graph which shows and explains the distribution of probability in a way that allows for the proficient dissemination of probability as well as an accurate representation [44], [53]. 
The nodes and edges of the graph indicate different things, and are interconnected in that group of random variables will dictate the system's nodes and these nodes are linked by a collection of edges. In terms of definitions, edges usually represent the reliance linking the cause and effect of statistical concepts, the latter being represented by nodes. The nodes at the highest levels can be understood as representing causes, while the final nodes are recognised as information taken in from the surroundings, or facts [53]. The influence of parents on each node, in other words, the parents' and nodes' probabilistic reliance on each other, is calculated by a conditional probability table that every node has. The probability that a pupil will complete a certain activity can be quantified by the DAG [53]. In circumstances where there is no parent present, the conditional probability table's result will consist of the connected random variable's probability value [53]. Bayesian Networks of great importance can be built using information from a variety of sources, however the function of categorizing users is one that BNs are particularly suited to in that they permit the fundamental characteristics of various users to be used as a basis for categorization [89].

There are a number of systems which have used Bayesian networks in adaptive educational system; the OLE system of Martn \&VanLehen [91], the ANDES system developed by [45], as well as the method proposed by Garca, Amandi, Schiaffino and Campo [92] which analyses the students" learning methods. Martn and VanLehen [91] developed OLE system and its purpose is student model assessment using Bayesian networks to observe the student's behaviour and then computing the probabilities of student knowledge. The work in [45] developed the ANDES system to find former probabilities of knowing a set of knowledge elemental parts. In contrast to these systems, [53] shows how the Bayesian networks are employed in the same manner to take users ' acquired knowledge and then make choices based on this information to identify which material is more suitable for particular students.

The approach that [53] proposed is based on Bayesian Networks and is utilized in determining the profile of the students according to the activities that they select. From this, it is possible to create the profile of the learner, and thus enable the learner envision their development is a particular subject. It also becomes possible for teachers to access students' profiles, which can be beneficial in enabling them devise the activities that each student ought to undertake or establish groups of students having related or similar attributes. Furthermore, the Bayesian Networks can assist in enhancing the presently-used systems and self-learning models. The rest of the examples of implementing Bayesian networks in adaptive educational system can be found in [93], [94], [95].

Indeed, acquiring the student knowledge and model by using Bayesian network consider to be NP-hard problem due to the dynamism of such knowledge and behavior in the learning environment[56].Therefore, such methods only work when applied in particular contexts [56]. In other words, the main problem highlighted with $\mathrm{BN}$ in practice is that the behaviors and varying levels of knowledge and understanding are too dynamic to properly be able to map, and as a result of this BN techniques are ineffective unless in specific and conditional situations [56].

\subsubsection{Hidden Markov Model}

A hidden Markov model (HMM) is a collection of unobserved states which follow the rules of the Markov property in a situation where the interrelationship between the true observation and an unobserved state takes birth from a probability distribution. In a nut shell HMM can be described as a probabilistic function related to an unobserved Markov chain [96],[97]. To be more accurate, a set of discrete states are described with the probability matrix being the determining factor of the transition in between the states. The pre- requisite of Markov property is that the model's state at time $t$ should be only determined by the model's state at time $t-1$ [97]. Any state before this should not play a role. The extra requirement of HMM is that the chances of observation of a symbol at time $t$ should be dependent on the state of model at time $t$. Rabiner and Juang [98] can be studied for obtaining a proper definition and extra facts about HMM. Greater research is now available about the various applications of HMM and they are being used in different ways like protein sequence modeling, identification of speech, multiple sequence alignment, searching of profiles and detection of regulatory sites [99]. 
It is possible to customize the system for an individual using the student behaviour prediction. The work presented in [100] provides a method that can be utilized to predict student's behaviour through the Markov hidden model. The input to the model constitutes of the actions that the user performed while training on how to interact with the electronic system. The pre-processing of the data acquired from the e-learning system informed the adjustments on the parameters of the Markov hidden models. The utilization of the Markov hidden models, by using the Baum Welch algorithm utilizing the training data and forward algorithms consequently, is used to predict the behaviour that the new user is likely to exhibit. In [101], Huang et al. presented the prediction of actions that students undertake afterwards through the weighted Markov model. The low-order Markov models have lowaccuracy levels while the high-order Markov models exhibit computational complexity. The weighting method was used to resolve the expressed problems. The weights exhibited the similarity between the actions that students undertake in the study and those of the students that are familiar with the system. In calculating the similarity, the sequence of actions done by each student is first demonstrated after which dynamic programming techniques are used to establish the share that is the largest between two learning paths and in calculating the consistency that the students' learning path have with the largest share [101].

\subsubsection{Genetic algorithms}

The use of genetic algorithms is particularly useful when it comes to understanding the preferences, wants and needs of the end user and as a result of this it has become increasingly popular for use in the education system [86]. The reason why genetic programming is such a helpful tool is primarily due to its processes and the way in which it attains information, it conducts a global search and is more adaptive when it comes to understanding the results.

This search algorithm uses the Darwin's concept of evolution, natural selection and survival of the fittest as its foundation and the basis for its process [86]. This process enables the computer software to identify the most relevant candidate(s) for the task at hand [86]. Using the process, the com- puter software gains the ability to establish the candidates that are most appropriate for the task being addressed [86]. In [102], an adaptive system was developed to provide the pedagogical paths adapted to the student profile as well as to the pedagogical objective of the existing formation. The problem is examined as an "Optimization Problem". By utilizing Genetic Algorithms, the focus of the system is to establish an optimal path beginning with the learner profile and extending to the pedagogical objective based on the intermediate courses. In [83], a personalized approach was presented for curriculum generation which is informed by a GA-based module to facilitate a personalized generation of a learning path as well as a CBR-based module for establishing a personalized knowledge database and conducting comprehensive assessment analysis. It is possible for the proposed approach of learning path generation to simultaneously incorporate the level of curriculum difficulty and the continuity of various curriculums while conducting curriculum development tailored to individuals in the process of learning

\section{Conclusion}

Adaptive educational systems emphasize that learning processes differ among learners. To ensure that study materials and e-learning services are tailor-made for adaptive learning, this particular educational approach attempts to integrate a capacity to diagnose the specific needs of each individual for the necessary learning. Such an approach facilitates the development of a suitable learning pedagogy while providing the most appropriate content to enhance the learning process. It therefore becomes crucial to establish accurate student models and profiles by modeling the affective states, personality traits, skills, levels, and level of knowledge of the various students. It is essential to use the acquired data efficiently to develop an adaptive learning environment. Acquired learner models can be used is two ways. The first is to inform the pedagogy proposed by designers and experts of adaptive educational system. The second is to provide the system with dynamic learning capacities from the behaviors exhibited by the teachers and students to create the appropriate pedagogy, while automatically adjusting the learning environments. In re- 
lation to this, artificial intelligence techniques can be very helpful, as they can develop and imitate the process of human reasoning and decision-making in designing the learning-teaching framework. Furthermore, they can handle uncertainty and facilitate the development of a context that promotes effective learning and teaching. Such learning capabilities are crucial in ensuring that both learners and the system improve over a sustained learning mechanism. This paper reviewed a number of emergent topics linked to how artificial intelligence techniques are used in adaptive educational systems. Furthermore, the paper presented the advantages of these techniques and systems and their significance in enhancing learning.

\section{References}

[1] L. A. James, Evaluation of an Adaptive Learning Technology as a Predictor of Student Performance in Undergraduate Biology, (Master's thesis), Appalachian State University, North Carolina, USA, May 2012.

[2] A.Ohle, N. McElvany, Teachers' diagnostic competences and their practical relevance. Special Issue Editorial, Journal for Educational Research Online, vol. 7, no. 2, 2015.

[3] B. Bloom, The 2 sigma problem: The search for methods of group instruction as effective as one-toone tutoring, Educ. Res., vol. 13, pp. 4-16, 1984.

[4] T. Kidd, Online Education and Adult Learning. New York: Hershey, 2010.

[5] M. Vandewaetere, P. Desmet, and G. Clarebout, The contribution of learner characteristics in the development of computer-based adaptive learning environments, Computers in Human Behavior, vol. 27, No. 1, pp. 118-130, 2011.

[6] C. Zhao, and L. Wan, A shortest learning path selection algorithm in e-learning, in Proc. 6th IEEE Int'1. Conf. on Advanced Learning Technologies (ICALT), 94-95, 2006.

[7] I. E. Allen, and J. Seaman, Staying the Course: Online Education in the United States. Sloan-C, Needham, MA: Sloan Consortium, 2008.

[8] Ambient Insight Research, US Self-Paced eLearning Market. Monroe, WA: Ambient Insight Research, 2009.

[9] S. Adkins, The Worldwide Market for Self-paced eLearning Products and Services: 2011-2016 Forecast and Analysis, Ambient Insight Premium Report, 2013.
[10] D. Ryan, E - learning Modules: DLR Associates Series, Author House, 2012

[11] R.C. Clark and R.E. Mayer, E-Learning and the Science of Instruction: Proven Guidelines for Consumers and Designers of Multimedia Learning, 3rd ed., San Francisco, USA: John Wiley \& Sons, 2011.

[12] H. Beetham and R.Sharpe, Rethinking pedagogy for a digital age:Designing for 21st century learning, New York, NY:Routledge, 2013.

[13] S.Selvakumarasamy and D.Dekson, "Architecture of Adaptive E-Learning Ecosystem, International Journal of Emerging Trends \& Technology in Computer Science (IJETTCS), 2013.

[14] B. Ciloglugil, and M. Inceoglu, User modeling for adaptive e-learning systems, Computational Science and Its Applications (ICCSA 2012), vol. 7335, pp. 5561, 2012.

[15] F. Essalmi, L. J. B. Ayed, M. Jemni, Kinshuk, and S. Graf, A fully personalization strategy of Elearning scenarios, Computers in Human Behavior, vol. 26, no. 4, pp. 581-591, 2010.

[16] Image from http://languageteachingtips.files.word press.com/2013/03/learningdeliverycontinuum.jpg, Retrieved Feburary 8, 2016

[17] V. J. Shute, and D. Zapata-Rivera, Adaptive educational systems, in P. Durlach (Ed.), Adaptive Technologies for Training and Education (pp. 7-27). New York: Cambridge University Press, 2012.

[18] S. Oxman, and W. Wong, White paper: Adaptive Learning Systems, A white paper from DVX/ DeVry Education Group and Integrated Education Solutions, 2014.

[19] I. Adaptive Learning, White paper based upon the Speak Up 2011 national findings, Leveraging Intelligent Adaptive Learning to Personalize Education, Intelligent Adaptive Learning: Speak Up Reports, 2012.

[20] C. Martins, L. Faria, and E. Carrapatoso, An adaptive educational system for higher education, Proceedings of the 14th EUNIS 08 International Conference of European University Information Systems, Denmark, 2008.

[21] S. Haggard, The maturing of the MOOC: Literature review of Massive Open Online Courses and other forms of Online Distance Learning (BIS Research Paper Number 130), Department for Business, Innovation and Skills, london uk, Research Papers Research paper number 130, 2013.

[22] X. Chen, D. Barnett, and C. Stephens, Fad or future: The advantages and challenges of massive 
open online courses (MOOCs), In Research-to Practice Conference in Adult and Higher Education, pp. 20-21, 2013.

[23] D. Onah, J. Sinclair, R. Boyatt (2014), Dropout rates of massive open online courses: behavioural patterns. Proceedings of the 6th International Conference on Education and New Learning Technologies, pp. 5825-5834, 2014.

[24] H. Fournier, R. Kop, and H. Sitlia, The Value of Learning Analytics to Networked Learning on a Personal Learning Environment, in Proceedings of the 1st International Conference on Learning Analytics and Knowledge, New York, NY, USA, 2011, pp. 104-109.

[25] S. Fauvel and H. Yu, A Survey on Artificial Intelligence and Data Mining for MOOCs, SciRate, 2016.

[26] L. C. Lin and . A. J. Prez, Educational Data Mining and Learning Analytics: differences, similarities, and time evolution," Rev. Univ. Soc. Conoc., vol. 12, no. 3, pp. 98-112, 2015.

[27] G. Siemens and R. S. J. d. Baker, Learning Analytics and Educational Data Mining: Towards Communication and Collaboration, in Proceedings of the 2Nd International Conference on Learning Analytics and Knowledge, New York, NY, USA, 2012, pp. 252-254.

[28] T. Daradoumis Haralabus, J. Faulin, . A. Juan Prez, F. J. Martnez, I. Rodrguez-Ardura, and F. Xhafa, Customer Relationship Management applied to higher education: developing an e-monitoring system to improve relationships in electronic learning environments, International Journal of Services Technology and Management, vol.14(1), pp. 103$125,2010$.

[29] T. Daradoumis, A. A. Juan, F. Lera-Lpez, and J. Faulin, Using Collaboration Strategies to Support the Monitoring of Online Collaborative Learning Activity, in Technology Enhanced Learning. Quality of Teaching and Educational Reform, M. D. Lytras, P. O. D. Pablos, D. Avison, J. Sipior, Q. Jin, W. Leal, L. Uden, M. Thomas, S. Cervai, and D. Horner, Eds. Springer Berlin Heidelberg, 2010, pp. 271-277.

[30] A. A. Juan, T. Daradoumis, J. Faulin, and F. Xhafa, A data analysis model based on control charts to monitor online learning processes, Int. J. Bus. Intell. Data Min., vol. 4, no. 2, pp. 159-174, 2009.

[31] B. R. Prakash, D. M. Hanumanthappa, and V. Kavitha, Big Data in Educational Data Mining and Learning Analytics, Int. J. Innov. Res. Comput. Commun. Eng, Vol. 2, Issue 12, 2014

[32] C. Romero and S. Ventura, Data Mining in Education, Wiley Int Rev Data Min Knowl Disc, vol. 3, no. 1, pp. 12-27, 2013.
[33] C. Romero and S. Ventura, Educational data mining: A survey from 1995 to 2005, Expert Syst. Appl., vol. 33, no. 1, pp. 135-146, 2007.

[34] C. Romero and S. Ventura, Educational Data Mining: A Review of the State of the Art, IEEE Trans. Syst. Man Cybern. Part C Appl. Rev., vol. 40, no. 6, pp. 601-618, 2010.

[35] A. Pea-Ayala, Review: Educational Data Mining: A Survey and a Data Mining-based Analysis of Recent Works, Expert Syst Appl, vol. 41, no. 4, pp. 1432-1462, 2014.

[36] A. M and Z. R. A. M. J. Md, A Comprehensive Survey on Educational Data Mining and Use of Data Mining Techniques for Improving Teaching and Predicting Student Performance, 2015, pp. 59-88

[37] R. Baker, Data Mining for Education, In: McGaw B, Peterson P, Baker E(eds) International Encyclopedia of Education (3rd edition), 7:112-118. Oxford, UK: Elsevier, 2010.

[38] R. Agrawal, T. Imielinski, and A. Swami, Mining Association Rules between Sets of Items in Large Databases, in: Proceedings Of The 1993 Acm Sigmod International Conference On Management Of Data, Washington DC (USA, 1993, pp. 207-216.

[39] E. Garca, C. Romero, S. Ventura, and T. Calders, Drawbacks and solutions of applying association rule mining in learning management systems, 2007, pp. 15-25.

[40] C. Romero, S. Ventura, M. Pechenizkiy, and R. S. J. d Baker, Handbook of Educational Data Mining. CRC Press, 2010

[41] B. K. Baradwaj and S. Pal, Mining Educational Data to Analyze Students' Performance, ArXiv12013417 Cs, Jan. 2012.

[42] E. Frias-Martinez, G. Magoulas, S. Chen, and R Macredie, Recent soft computing approaches to user modeling in adaptive hypermedia, In Adaptive Hypermedia and Adaptive Web-Based Systems, the series Lecture Notes in Computer Science, vol. 3137, pp. 104-114, Springer Berlin Heidelberg, 2004.

[43] FM. Cin, and AF. Baba, Assessment of English proficiency by fuzzy logic approach, In International Educational Technology Conference, pp. 355-359. 2008.

[44] H. Gamboa, Designing Intelligent Tutoring Systems : A Bayesian Approach, Proc. of Ana Fred 3rd International Conference on Enterprise Information Systems (ICEIS'2001), 2001, pp. 452-458.

[45] A. Gertner, and K.VanLehn, Andes: A coached problem solving environment for physics, In Intelligent Tutoring Systems, vol.1839, pp. 133-142, Springer Berlin Heidelberg, 2000. 
[46] J. Ma and D. N. Zhou, Fuzzy set approach to the assessment of student centered learning, IEEE Trans. Educ., vol. 33, pp. 237-241, 2000.

[47] H. Seridi-Bouchelaghem, T. Sari, and M. Sellami, A Neural Network for Generating Adaptive Lessons, Journal of Computer Science 1, no. 2, pp. 232-243, 2005.

[48] R. Sripan and B. Suksawat, Propose of Fuzzy Logic-Based Students' Learning Assessment, Proceedings in the International Conference on Control, Automation and Systems, pp. 414-417, Gyeonggido, Korea, 2010.

[49] S. Venkatesan, and S. Fragomeni, Evaluating learning outcomes in PBL using fuzzy logic techniques, 19th Annual Conference of the Australasian Association for Engineering Education: To Industry and Beyond; Proceedings of the. Institution of Engineers, Australia, 2008.

[50] H. Cha, Y. Kim, S. Park, T. Yoon, Y. Jung, and J. Lee, Learning Style Diagnosis Based on User Interface Behavior for the Customization of Learning Interfaces in an Intelligent Tutoring System, Proceedings of the 8th International Conference on Intelligent Tutoring Systems, the series Lecture Notes in Computer Science, vol. 4053, pp. 513-524, Springer Berlin Heidelberg, 2006.

[51] N. Idris, N. Yusof, and P. Saad, Adaptive course sequencing for personalization of learning path using neural network, International Journal of Advanced Soft Computing and Its Applications, vol. 1, no.1, pp. 49-61, 2009.

[52] S. Gutierrez-Santos, J. Mayor-Berzal, C. Fernandez-Panadero, and CR. Kloos, Authoring of Probabilistic Sequencing in Adaptive Hypermedia with Bayesian Networks, Journal of Universal Computer Science, vol.16, no. 19, pp. 2801-2820, 2010.

[53] F. Moreno, A. Carreras, M. Moreno and E. R. Royo, Using Bayesian Networks in the Global Adaptive E-learning Process, EUNIS 2005, Manchester, pp. 1-4, 2005.

[54] D. Xu, H. Wang and K. Su, Intelligent student profiling with fuzzy models, in Proceedings of the 35th Hawaii International Conference on System Science (HICSS 2002), Hawaii, U.S.A, 2002.

[55] P. Brusilovsky, and E. Milln. User models for adaptive hypermedia and adaptive educational systems, The Adaptive Web, the series Lecture Notes in Computer Science, vol. 4321, pp. 3-53, Springer Berlin Heidelberg, 2007.

[56] A. Ahmad, O. Basir, and K. Hassanein, Adaptive user interfaces for intelligent e-Learning: issues and trends, in Proceedings of the Fourth International Conference on Electronic Business (ICEB2004), Xiyuan Hotel, Beijing, China, pp. 925-934, 2004.

[57] L. A. Zadeh, Fuzzy sets, Inf. Control, vol. 8, pp. 338-353, 1965.

[58] J. Bih, Paradigm shift -an introduction to fuzzy logic, IEEE Potentials, vol. 25, no. 1, pp. 6-21, 2006.

[59] J. Mendel, Fuzzy logic system for engineering: A tutorial, Proceedings of the IEEE, 1995, vol. 83, no. 3, pp. 345-374.

[60] J. Mendel, Uncertain Rule-Based Fuzzy Logic Systems, Prentice Hall 2001.

[61] J. Mendel, H. Hagras, W. Tan, W. Melek, and H. Ying, Introduction to Type-2 Fuzzy Logic Control, John Wiley and IEEE Press,Hoboken, NJ, 2014

[62] A. Jameson, Numerical uncertainty management in user and student modeling: An overview of systems and issues, Use Modeling and User-adapted Interaction, vol. 5(3-4), pp. 103-251, 1996.

[63] A. Kavi, R. Pedraza-Jimnez, H. Molina-Bulla, F.J. Valverde-Albacete, J. Cid-Sueiro, and A. NaviaVzquez, Student Modelling Based on Fuzzy Inference Mechanisms, Proceedings of the IEEE Region 8 EUROCON 2003, Computer as a Tool, Ljubljana, Slovenia, 2003.

[64] D. Chang, and C. Sun (1993), Fuzzy Assessment Learning Performance of Junior High School Students, Proceedings of the 1993 First National Symposium on Fuzzy Theory and Applications, Hsinchu, Taiwan, Republic of China, pp. 1-10, 1993.

[65] S. Chen, and C. Lee, New methods for students' evaluating using fuzzy sets, Fuzzy Sets and Systems, vol.104, pp. 209-218, 1999.

[66] O. Nyknen, Inducing fuzzy models for student classification, Journal of Educational Technology and Society, vol.9, pp. 223-234,2006.

[67] S. Weon, J. Kim, Learning achievement evaluation strategy using fuzzy membership function, In Proceedings of the 31st ASEE/IEEE Frontiers In Education Conference, Reno, NV (pp. 19-24),2001.

[68] A. Kavi, Fuzzy user modeling for adaptation in educational hypermedia, IEEE Transactions on Systems, Man, and Cybernetics, Part C: Applications and Reviews, vol. 34, no. 4, pp. 439-449, 2004.

[69] K. Almohammadi and H. Hagras, An Interval Type-2 Fuzzy Logic Based System for Customised Knowledge Delivery within Pervasive E-Learning Platforms, Proceeings of the 2013 IEEE International Conference on Systems, Man, and Cybernetics, pp. 2872-2879, 2013. 
[70] K. Almohammadi and H. Hagras, An adaptive fuzzy logic based system for improved knowledge delivery within intelligent E-Learning platforms, Proccedings of the the 2013 IEEE International Conference on Fuzzy Systems (FUZZ-IEEE), pp. 18, 2013.

[71] K. Almohammadi, B. Yao, and H. Hagras, An interval type-2 fuzzy logic based system with user engagement feedback for customized knowledge delivery within intelligent E-learning platforms, Proceedings of the 2014 IEEE International Conference on Fuzzy Systems (FUZZ-IEEE), pp. 808-817, 2014.

[72] K. Almohammadi, H. Hagras, B. Yao, A. Alzahrani, D.Alghazzawi, and G. Aldabbagh, A Type-2 Fuzzy Logic Recommendation System for Adaptive Teaching, Journal of Soft Computing, 2015.

[73] T. M. Lakshmi, A. Martin, R. M. Begum, and V. P. Venkatesan, An Analysis on Performance of Decision Tree Algorithms using Student's Qualitative Data, I.J.Modern Education and Computer Science, vol. 5, pp. 18-27, 2013.

[74] Margret H. Dunham, Data Mining: Introductory and advance topic, Pearson Education India, 2006.

[75] J.R.Quinlan, Induction of Decision Tree, Journal of Machine learning, Morgan Kaufmann Vol.1, 1986, pp. 81-106.

[76] C.-M. Chen, Intelligent Web-based Learning System with Personalized Learning Path Guidance, Comput. Educ., vol. 51, no. 2, pp. 787-814, 2008.

[77] C. F. Lin, Y.-C. Yeh, Y. H. Hung, and R. I. Chang, Data Mining for Providing a Personalized Learning Path in Creativity: An Application of Decision Trees, Comput Educ, vol. 68, pp. 199-210, 2013.

[78] B. Kumar Baradwaj, S. Pal, Mining Educational Data to Analyze Students' Performance, International Journal of Advanced Computer Science and Applications, Volume 2, No. 6, 2011, pp 63-69.

[79] Adhatrao K, Gaykar A, Dhawan A, Jha R, Honrao V, Predicting Students' Performance using ID3 and C4.5 classification algorithms,International Journal of Data Mining \& Knowledge Management Process, Volume 3, No. 5, pp 39-52, 2013, DOI :10.5121/ijdkp.2013.3504.

[80] M. Pandey, V. Kumar Sharma, A Decision Tree Algorithm Pertaining to the Student Performance Analaysis and Prediction, International Journal of Computer Applications, volume 61, No. 13, Jan 2013, pp 1-5, DOI 10.5120/9985-4822
[81] B. K. Baradwaj and S. Pal, Mining Educational Data to Analyze Students' Performance, ArXiv12013417 Cs, 2012.

[82] Giarratano, J., \& Riley, G, Expert systemsprinciple and programming, (3rd ed.). Boston, MA: PWS Publishing Company, 1998.

[83] M. Huang, H. Huang, and M. Chen, Constructing a personalized e-learning system based on genetic algorithm and case-based reasoning approach, Expert Syst. Appl., vol. 33, no. 3, pp. 551-564, Oct. 2007.

[84] L. Fausett, Ed., Fundamentals of Neural Networks: Architectures, Algorithms, and Applications. Upper Saddle River, NJ, USA, Prentice-Hall, Inc., 1994.

[85] S. Haykin, Neural Networks: A Comprehensive Foundation, 2nd ed. Upper Saddle River, NJ, USA: Prentice Hall PTR, 1998.

[86] A. S. Drigas, K. Argyri, and J. Vrettaros, Decade Review, Artificial Intelligence Techniques in Student Modeling, in Best Practices for the Knowledge Society. Knowledge, Learning, Development and Technology for All, vol. 49, M. D. Lytras, P. Ordonez de Pablos, E. Damiani, D. Avison, A. Naeve, and D. G. Horner, Eds. Berlin, Heidelberg: Springer Berlin Heidelberg, 2009, pp. 552-564.

[87] B. Naik and S. Ragothaman, Using Neural Networks to Predict MBA Student Success, Coll. Stud. J., vol. 38, no. 1, p. 143, 2004.

[88] Z. Ibrahim and D. Rusli, Predicting students' academic performance: comparing artificial neural network, decision tree and linear regression, in 21st Annual SAS Malaysia Forum, 2007.

[89] C. Gonzalez, J. Burguillo, and M. Llamas, A Qualitative Comparison of Techniques for Student Modeling in Intelligent Tutoring Systems, 2006, pp. 1318.

[90] Martn, J., VanLehn, K. OLAE: Progress toward a multi-activity,Bayesian student modeler. Proceedings of the World Conference on Artificial Intelligence in Education. 1993, pp. 410-417.

[91] P. Garca, A. Amandi, S. Schiaffino, and M. Campo, Using Bayesian networks to detect students' learning styles in a web-based education system, Proc ASAI Rosario, pp. 115-126, 2005.

[92] C. Conati, A. Gertner, and K. Vanlehn, Using Bayesian networks to manage uncertainty in student modeling, User Model. User-Adapt. Interact., vol. 12, no. 4, pp. 371-417, 2002.

[93] V. J. Shute, E. G. Hansen, and R. G. Almond, You Can't Fatten A Hog by Weighing It - Or Can You? Evaluating an Assessment for Learning System Called ACED, Int. J. Artif. Intell. Educ., vol. 18, no. 4, pp. 289-316, 2008. 
[94] K. Vanlehn, C. Lynch, K. Schulze, J. A. Shapiro, R. Shelby, L. Taylor, D. Treacy, A. Weinstein, and M. Wintersgill, The Andes Physics Tutoring System: Lessons Learned,Int. J. Artif. Intell. Ed, vol. 15, no. 3, pp. 147-204, 2005.

[95] C. J. Burke and M. Rosenblatt. A markovian function of a markov chain. The Annals of Mathematical Statistics, Vol. 29, No. 4, pp. 1112-1122, 1958.

[96] B. Shih, K. R. Koedinger, and R. Scheines, Discovery of Student Strategies using Hidden Markov Model Clustering, the Proceedings of the 6th International Conference on Educational Data Mining. 2010 .

[97] L. Rabiner and B. Juang, An introduction to hidden Markov models, IEEE ASSP Mag., vol. 3, no. 1, pp. 4-16, 1986

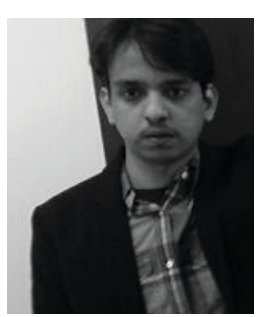

Khalid Almohammadi completed his bachelor's degree in computer science from Taibah University in the Kingdom of Saudi Arabia. He then pursued a profession in teaching at Tabuk University. Building on this experience, he was awarded a scholarship to complete his master's in computer science (MSc) at Newcastle University in England, which he obtained with distinction in 2011. He is currently in the process of completing his $\mathrm{PhD}$ in computer science at Essex University in England, which incorporates all his previous knowledge and experience in education and e-learning. The focus of his research is the development of theoretical and practical environments to enhance the learning experience and engagement by using fuzzy logic systems

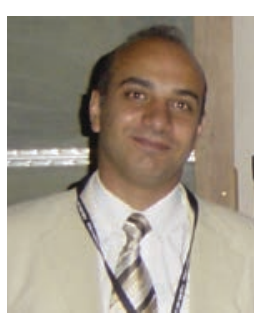

Hani Hagras received the B.Sc. and M.Sc. degrees in electrical engineering from Alexandria University, Alexandria, Egypt, and the Ph.D. degree in computer science from the University of Essex, Colchester, U.K. He is a Professor in the School of Computer Science and Electronic Engineering, Director of the Computational Intelligence Centre and the Head of the Fuzzy Systems Research Group in the University of Essex, UK. His major research interests are in computational intelligence, notably type-2 fuzzy systems, fuzzy logic, neural networks, genetic algorithms, and evolutionary computation. His research interests also include ambient intelligence, pervasive computing and intelligent buildings. He is also interested in embedded agents, robotics and intelligent control. He has authored more than 300 papers in international journals, conferences and books. He is a Fellow of the Institute of Electrical and Electronics Engineers (IEEE) and he is also a Fellow of the Institution of Engineering and Technology (IET (IEE). He was the Chair of IEEE Computational Intelligence Society (CIS) Senior Members Sub-Committee. His research has won nu-
[98] T. Doleck, R. B. Basnet, E. G. Poitras, and S. P. Lajoie, Mining learner-system interaction data: implications for modeling learner behaviors and improving overlay models, J. Comput. Educ., vol. 2, no. 4, pp. 421-447, Aug. 2015.

[99] Morteza.S. Anari, Maryam. S. Anari, Intelligent ELearning Systems Using Student Behavior Prediction, J. Basic. Appl. Sci. Res., 2(12)12017-12023, 2012.

[100] X. Huang, J. Yong, J. Li, J. Gao, Prediction of student actions using weighted Markov models, IT in Medicine and Education, IEEE International Symposium on Digital Object Identifier, 2008.

[101] S. Azough and M. B. E. H. Bouyakhf, Adaptive E-learning using Genetic Algorithms, IJCSNS Int. J. Comput. Sci. Netw. Secur., vol. 10, no. 7, pp. 237 277, 2010. merous prestigious international awards where most recently he was awarded by the IEEE Computational Intelligence Society (CIS), the 2013 Outstanding Paper Award in the IEEE Transactions on Fuzzy Systems and he was also awarded the 2006 Outstanding Paper Award in the IEEE Transactions on Fuzzy Systems. He is an Associate Editor of the IEEE Transactions on Fuzzy Systems. He is also an Associate Editor of the International Journal of Robotics and Automation, the Journal of Cognitive Computation and the Journal of Ambient Computing and Intelligence. He is a member of the IEEE Computational Intelligence Society (CIS) Fuzzy Systems Technical Committee and IEEE CIS conference committee. Prof. Hagras chaired several international conferences where he served as the General Co-Chair of the 2007 IEEE International Conference on Fuzzy Systems London.

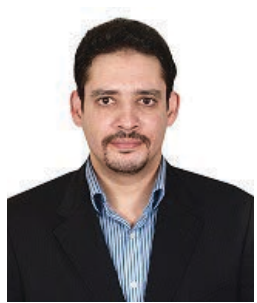

Daniyal Alghazzawi obtained his Bachelor's degree with honor in Computer Science from King Abdulaziz University (KAU) in 1999. He completed his master's degree and doctorate in the field of Computer Science at the University of Kansas at the United States in 2007. He also obtained another master's degree in Teaching and Leadership from University of Kansas in 2004. He also obtained the certificate of Management International Leadership (LMI) and has been the Head of the Information Systems department, Faculty of Computing and Information Technology for over five years during which he organized many workshops, and international and domestic conferences. He is currently an Associate Professor in the Department of Information Systems, Faculty of Computing I\& Information Technology at King Abdulaziz University. He is also the head of the Information Security Research Group at King Abdulaziz. He has published 60 papers in various international journals, conferences and books in the field of Intelligent Systems and Information Security. His research interests include intelligent environments, computational intelligence, smart e-learning and information security. 


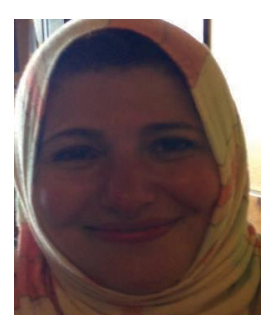

Ghadah Aldabbagh received her BSc in 1991 in Electrical Engineering (Computer Engineering Specialization) from the University of Illinois at Urbana - Champaign, Illinois, United States; received her MSc in the Data Communication Networks and Distributed System (DCNDS) Program in the Computer Science Department, University College London (London), United Kingdom (UK); received her PhD in 2010 from the Department of Electronic and Electrical Engineering from the University College London (UCL), United Kingdom (UK). She holds an Assistant Professor position at the Faculty of Computing and Information Technology in the Computer Science Department at King Abdul Aziz University. Her research covers communication systems and networks, networking algorithms and performance evaluation and protocols and algorithms. 